\title{
CONSUMO DE CARNE DE RES DE LAS FAMILIAS AMBATEÑAS
}

\section{Juan Pablo Martínez Mesías1} Lilián Morales2
Fecha de recepción: 15 Noviembre 2016

Fecha de aceptación: 30 Diciembre 2016
Pag 4-6

\section{Resumen}

- ste artículo determina el perfil de consumo de carne de res Een las familias del cantón Ambato. Se empleó un cuestionario y se obtuvieron 403 respuestas en la zona urbana y rural. La cantidad adquirida es de 7 libras total en alrededor de 8 veces mensuales, gastando en promedio 44,62 USD. Los consumidores prefieren el empaque al vacío que normalmente adquieren en supermercados y plazas-mercados. Los datos contribuyen a la planificación comercial de los establecimientos destinados a la venta de carne, así como también en el diseño de políticas públicas en áreas de salud y comercialización.

\section{Palabras clave:}

Perfil de consumo, preferencia de compra, carne de res

\section{Abstract}

The purpose of this paper is to establish the meat consumption profile of Ambato's families. By using a questionnaire, 403 people from urban and rural zones have been surveyed. The investigation describes beef purchases preferences considering 3.4 people per family, beef consumption per family is on average of 7 pounds with a frequency of 8 times a month and the spending is around 44,62 USD. With regard to packaging, people prefer vacuum packaging, which are found commonly at markets and supermarkets. This information contributes to commercial planning as well as to public politics design concerning health and merchandizing.

\section{Keywords:}

Economic growth, manufactures, causation.

\section{Introducción}

El objetivo del artículo es establecer el perfil de consumo de carne de ganado vacuno sin procesar de las familias del cantón Ambato. La carne de res sin hueso es uno de los productos con mayor contribución al incremento del Índice de precios al consumo (IPC) de la Zona 3 del Ecuador, según los datos del Ministerio de Agricultura, Ganadería, Acuacultura y Pesca-MAGAP a diciembre del año 2015.

\section{Metodología}

Al no contar con datos oficiales sobre el consumo de carne de res por familia en el cantón Ambato, se realiza un estudio descriptivo mediante el cual se procede a recolectar información por medio de un formulario de encuesta, en base a los hábitos de consumo y perfil del consumidor, aplicado el mes de mayo del año 2016 en todo el cantón Ambato de la provincia de Tungurahua. Para el estudio, se cuenta con una población 369.578 habitantes en el cantón Ambato al año 2016, en base a la Proyección de la Población Ecuatoriana por años calendario, según cantones 2010-2020 del Instituto Nacional de Estadística y Censos (INEC).

Con lo descrito, para la estimación de la muestra se aplicó la fórmula de población infinita con un error aceptado de 5\% y $a=.05$ ( $z=1.96)$, lo que da como resultado una muestra de 384 personas. Sin embargo, se recolectaron 403 respuestas que corresponden a las personas responsables de la compra familiar que residen en la zona urbana y rural de Ambato.

\section{Contenido}

El consumo de carne roja en el cantón Ambato

En cuanto al consumo de carne roja, los resultados muestran un consumo del $82,1 \%$ de carne de res en la población encuestada en el cantón Ambato y en menor proporción la carne de cerdo con 17,9\%.

\section{Figura 1. Perfil del consumo de carne de res de las familias ambateñas}

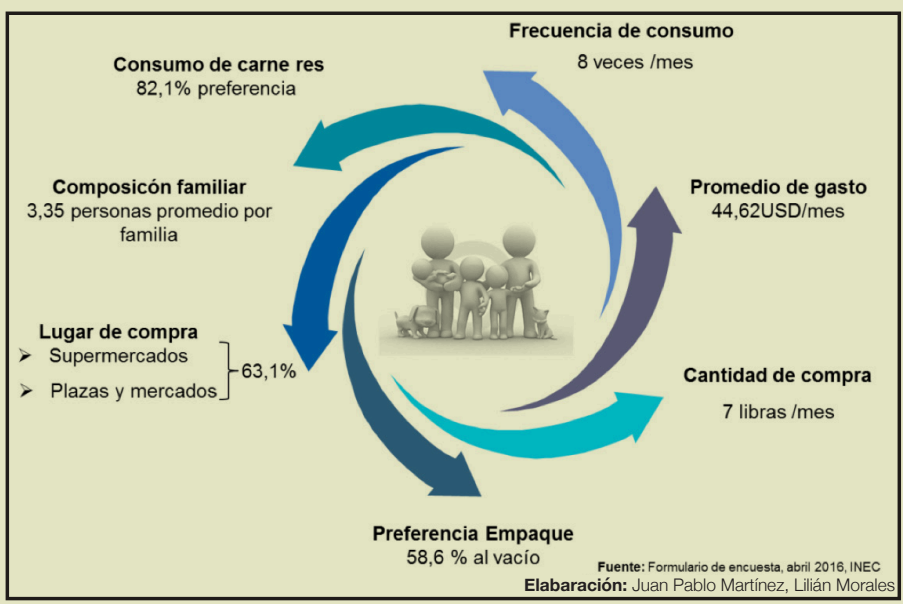

En la Tabla 1 se presenta la información en cuanto a la compra semanal de carne de res en cantidad (libras) y gasto (dólares). Estos resultados muestran que las familias ambateñas compran en términos absolutos 2 libras semana, en promedio, mientras que el gasto/semana se concentra entre los rangos de "6USD a 10USD" y "11USD a 15USD" con un peso de 70,1\%. Si extrapolamos los resultados a la compra mensual de carne res, resulta que las familias ambateñas compran en promedio 7 libras de carne de res al mes, con un gasto medio mensual de 44,62 USD.

Tabla 1. Compra por semana de carne de res en el cantón Ambato

\begin{tabular}{|c|c|c|c|c|c|}
\hline \multicolumn{3}{|c|}{ Compra por semana de carne de res } & \multicolumn{3}{|c|}{ Gasto en dólares por semana en carne de res } \\
\hline Cantidad & Frecuencia & Porcentaje & $\begin{array}{l}\text { Gasto en } \\
\text { dólares }\end{array}$ & Frecuencia & Porcentaje \\
\hline Menos de 2 libras & 209 & $63 \%$ & Hasta 5\$ & 24 & $7,3 \%$ \\
\hline 2- 4 libras & 102 & $31 \%$ & $6 \$-10 \$$ & 132 & $39,9 \%$ \\
\hline \multirow[t]{3}{*}{ Más de 4 libras } & 20 & $6 \%$ & $11 \$-15 \$$ & 100 & $30,2 \%$ \\
\hline & & & $16 \$-20 \$$ & 59 & $17,8 \%$ \\
\hline & & & Más de 20\$ & 16 & $4,8 \%$ \\
\hline Total & 331 & $100 \%$ & Total & 331 & $100,0 \%$ \\
\hline \multicolumn{6}{|c|}{$\begin{array}{l}\text { Fuente: Formulario de encuesta abril } 2016 \\
\text { Elabaración: Juan Pablo Martínez, Lilián Morales }\end{array}$} \\
\hline
\end{tabular}

Al analizar el consumo de carne de res, se obtiene una frecuencia promedia de dos veces/semana, siendo esta opción la más representativa para $47 \%$ de los encuestados. En términos mensuales la frecuencia de consumo es de 8 veces.

Economista, Magíster en Administración de Empresas mención Planeación - Docente de la Facultad de Contabilidad y Auditoría - jpmartinez@uta.edu.ec

${ }^{2}$ Doctora en Ciencias Económicas y Empresariales - Docente de la Facultad de Contabilidad y Auditoría - lilianmorales@uta.edu.ec 
Figura 2. Frecuencia consumo carne de res por semana de las familias Ambato

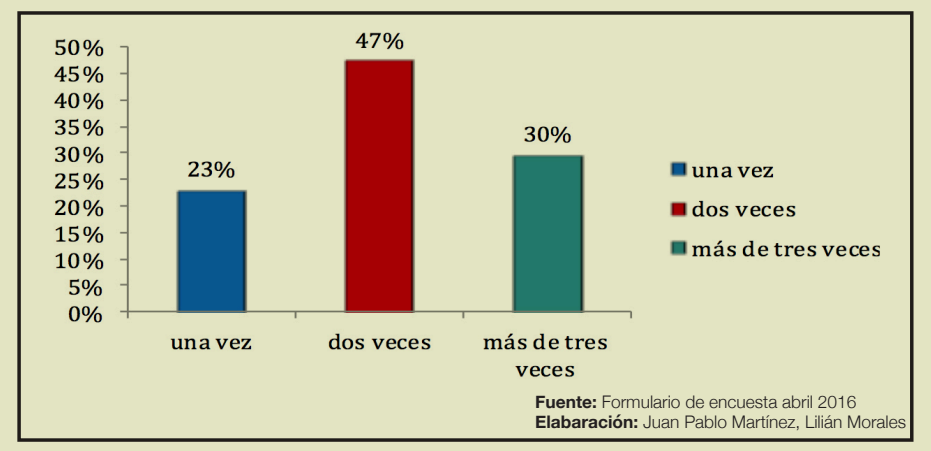

Otras de las características estudiadas son: lugar de compra y presentación del producto. En relación al primer aspecto mencionado, las familias prefieren comprar la carne de res en supermercados $(34,4 \%)$ y en plazas/mercados (28,7\%). En referencia al segundo aspecto, se tiene que más de la mitad $(58,6 \%)$ elige comprar empacada al vacío.

Tabla 2. Cantidad de compra semanal carne de res, en libras por ingresos de las familias

\section{Ambato}

\begin{tabular}{|c|c|c|c|c|c|c|c|c|c|c|}
\hline \multirow{3}{*}{$\begin{array}{c}\text { Cantidad } \\
\text { compra en } \\
\text { libras }\end{array}$} & \multicolumn{8}{|c|}{ Ingreso } & \multirow{3}{*}{ Total } & \multirow{3}{*}{$\%$} \\
\hline & \multicolumn{2}{|c|}{$\begin{array}{c}\text { Menos de } \\
\$ 366\end{array}$} & \multicolumn{2}{|c|}{$\begin{array}{c}\text { Entre } \$ 366 \text { y } \\
\$ 500\end{array}$} & \multicolumn{2}{|c|}{$\begin{array}{c}\text { Entre } \$ 500 y \\
\$ 1000\end{array}$} & \multicolumn{2}{|c|}{ Más de $\$ 1000$} & & \\
\hline & Frec. & $\%$ & Frec. & $\%$ & Frec. & $\%$ & Frec. & $\%$ & & \\
\hline $\begin{array}{l}\text { Menos de } 2 \\
\text { libras }\end{array}$ & 36 & $17,2 \%$ & 62 & $29,7 \%$ & 88 & $42,1 \%$ & 23 & $11,0 \%$ & 209 & $63,1 \%$ \\
\hline 2-4 libras & 9 & $8,8 \%$ & 23 & $22,5 \%$ & 53 & $52,0 \%$ & 17 & $16,7 \%$ & 102 & $30,8 \%$ \\
\hline $\begin{array}{l}\text { Más de } 4 \\
\text { libras }\end{array}$ & 4 & $20,0 \%$ & 6 & $30,0 \%$ & 6 & $30,0 \%$ & 4 & $20,0 \%$ & 20 & $6,0 \%$ \\
\hline Total & 49 & $14,8 \%$ & 91 & $27,5 \%$ & 147 & $44,4 \%$ & 44 & $13,3 \%$ & 331 & $100,0 \%$ \\
\hline & & & & & & $\begin{array}{l}\text { Fuente: } \\
\text { Elabarac }\end{array}$ & $\begin{array}{l}\text { Formu } \\
\text { ción: }\end{array}$ & able & IE, & $\begin{array}{l}016 \\
0 \text { án Morales }\end{array}$ \\
\hline
\end{tabular}

Del total de los encuestados el 63,1\% compra en promedio menos de 2 libras de carne de res por semana; esta cantidad es comprada mayoritariamente por el segmento de ingreso entre $\$ 500$ a $\$ 1.000$ (42,1\%). Además el 30,8\% compra entre 2 y 4 libras de carne de res por semana y corresponde al mismo segmento de ingresos. Por lo tanto, el 93,9\% de familias del cantón Ambato, compran hasta 4 libras de carne de res a la semana.

Tabla 3. Cantidad de compra semanal en libras de carne de res, según estructura familiar

Ambato

\begin{tabular}{|c|c|c|c|c|c|c|}
\hline \multirow{3}{*}{$\begin{array}{c}\text { Cantidad } \\
\text { compra en } \\
\text { libras }\end{array}$} & \multicolumn{4}{|c|}{ Números Miembros Familiares } & \multirow{3}{*}{ Total } & \multirow{3}{*}{$\%$} \\
\hline & \multicolumn{2}{|c|}{ Hasta 3 miembros } & \multicolumn{2}{|c|}{ Más de 3 miembros } & & \\
\hline & Frec. & $\%$ & Frec. & $\%$ & & \\
\hline $\begin{array}{l}\text { Menos de } 2 \\
\text { libras }\end{array}$ & 132 & $63,2 \%$ & 77 & $36,8 \%$ & 209 & $63,1 \%$ \\
\hline 2 - 4 libras & 45 & $44,1 \%$ & 57 & $55,9 \%$ & 102 & $30,8 \%$ \\
\hline $\begin{array}{l}\text { Más de } 4 \\
\text { libras }\end{array}$ & 9 & $45,0 \%$ & 11 & $55,0 \%$ & 20 & $6,0 \%$ \\
\hline \multirow[t]{2}{*}{ Total } & 186 & $56,2 \%$ & 145 & $43,8 \%$ & 331 & $100 \%$ \\
\hline & & & \multicolumn{4}{|c|}{$\begin{array}{l}\text { Fuente: Formulario de encuesta abril } 2016 \\
\text { Elabaración: Juan Pablo Martínez, Lilián Morales }\end{array}$} \\
\hline
\end{tabular}

Los resultados de la encuesta arrojan que en el Cantón de Ambato hay un promedio de 3,35 personas por hogar, un dato muy cercano al promedio de 3,57 publicado por INEC. Del total de encuestados, el 56,2\% tienen a su cargo una familia de hasta 3 miembros, mientras que un 43,8\% corresponde a familias con más de 3 miembros, revelando este último segmento que compran menos 2 libras y de 2 hasta 4 libras de carne de res por semana. Por otro lado, quienes tienen a su cargo hasta 3 miembros familiares compran mayoritariamente menos de 2 libras de carne de res a la semana.

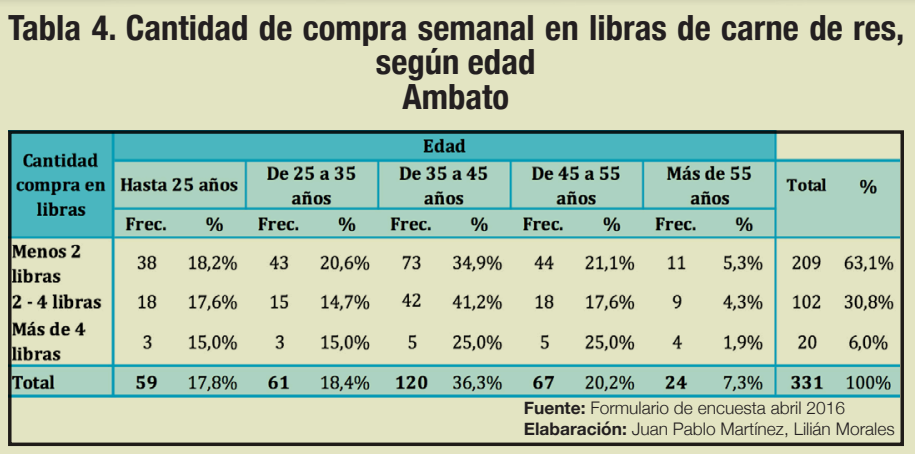

El $36,3 \%$ de los encuestados que han declarado que consumen carne de res se encuentran en el rango de edad de 35 a 45 años y un $20,2 \%$ de 45 a 55 años de edad. Es decir, un 56,5\% de la población encuestada que dice consumir carne de res está en el rango de edad de 35 a 55 años, con un hábito de compra promedio de menos de 2 libras de carne de res semanales.

Tabla 5. Compra semanal de carne de res en dólares, según ingreso de las familias

Ambato

\begin{tabular}{|c|c|c|c|c|c|c|c|c|c|c|}
\hline \multirow{3}{*}{$\begin{array}{l}\text { Compra en } \\
\text { dólares }\end{array}$} & \multicolumn{8}{|c|}{ Ingreso } & \multirow{3}{*}{ Total } & \multirow{3}{*}{$\%$} \\
\hline & \multicolumn{2}{|c|}{ Hasta $\$ 366$} & \multicolumn{2}{|c|}{$\$ 366-\$ 500$} & \multicolumn{2}{|c|}{$\$ 500-\$ 1000$} & \multicolumn{2}{|c|}{ Más de $\$ 1000$} & & \\
\hline & Frec. & $\%$ & Frec. & $\%$ & Frec. & $\%$ & Frec. & $\%$ & & \\
\hline Hasta 5\$ & 7 & $29,2 \%$ & 10 & $41,7 \%$ & 6 & $25,0 \%$ & 1 & $4,2 \%$ & 24 & $7,3 \%$ \\
\hline $6 \$-10 \$$ & 25 & $18,9 \%$ & 46 & $34,8 \%$ & 50 & $37,9 \%$ & 11 & $8,3 \%$ & 132 & $39,9 \%$ \\
\hline $11 \$-15 \$$ & 10 & $10,0 \%$ & 22 & $22,0 \%$ & 51 & $51,0 \%$ & 17 & $17,0 \%$ & 100 & $30,2 \%$ \\
\hline $16 \$-20 \$$ & 5 & $8,5 \%$ & 10 & $16,9 \%$ & 34 & $57,6 \%$ & 10 & $16,9 \%$ & 59 & $17,8 \%$ \\
\hline Más de $20 \$$ & 2 & $12,5 \%$ & 3 & $18,8 \%$ & 6 & $37,5 \%$ & 5 & $31,3 \%$ & 16 & $4,8 \%$ \\
\hline Total & 49 & $14,8 \%$ & 91 & $27,5 \%$ & 147 & $44,4 \%$ & 44 & $13,3 \%$ & 331 & $100 \%$ \\
\hline & & & & & & $\begin{array}{l}\text { Fuente } \\
\text { Elabare }\end{array}$ & $\begin{array}{l}\text { Formu } \\
\text { cción: J }\end{array}$ & $\begin{array}{l}\text { o de encu } \\
\text { n Pablo } \mathrm{N}\end{array}$ & tínez, & $\begin{array}{l}\text { 16 } \\
\text { in Morales }\end{array}$ \\
\hline
\end{tabular}

El 39,9\% de los encuestados realiza compras de carne de res a la semana de entre $\$ 6$ y $\$ 10$, seguidos por el rango de $\$ 11$ hasta $\$ 15$, con un 30,2\%. De las familias que compran en el rango de $\$ 6$ hasta $\$ 10$, el 37,9\% corresponde al segmento de ingreso de entre $\$ 500$ $\$ 1000$, seguido por el segmento de $\$ 360$ a $\$ 500$ con un $34,8 \%$. En lo que concierne a las compras de $\$ 11$ a $\$ 15$ semanales el $51 \%$ pertenece al segmento de ingresos de entre $\$ 500$ a $\$ 1.000$.

\section{Tabla 6. Compra semanal de carne de res en dólares, según estructura familiar \\ Ambato}

\begin{tabular}{|c|c|c|c|c|c|c|}
\hline \multirow{3}{*}{$\begin{array}{l}\text { Gasto en } \\
\text { dólares }\end{array}$} & \multicolumn{4}{|c|}{$\begin{array}{c}\text { Número de miembros } \\
\text { familiares }\end{array}$} & \multirow{3}{*}{ Total } & \multirow{3}{*}{$\%$} \\
\hline & \multicolumn{2}{|c|}{ Hasta 3} & \multicolumn{2}{|c|}{ Más de 3} & & \\
\hline & Frec. & $\%$ & Frec. & $\%$ & & \\
\hline Hasta 5\$ & 14 & $7,5 \%$ & 10 & $6,9 \%$ & 24 & $7,3 \%$ \\
\hline $6 \$-10 \$$ & 93 & $50,0 \%$ & 39 & $26,9 \%$ & 132 & $39,9 \%$ \\
\hline $11 \$-15 \$$ & 48 & $25,8 \%$ & 52 & $35,9 \%$ & 100 & $30,2 \%$ \\
\hline $16 \$-20 \$$ & 25 & $13,4 \%$ & 34 & $23,4 \%$ & 59 & $17,8 \%$ \\
\hline Más de 20\$ & 6 & $3,2 \%$ & 10 & $6,9 \%$ & 16 & $4,8 \%$ \\
\hline Total & 186 & $100 \%$ & 145 & $100 \%$ & 331 & $100 \%$ \\
\hline \multicolumn{7}{|c|}{$\begin{array}{l}\text { Fuente: Formulario de encuesta abril } 2016 \\
\text { Elabaración: Juan Pablo Martinezz, Lilián Morales }\end{array}$} \\
\hline
\end{tabular}


El 50\% de las familias de hasta 3 miembros gastan entre $\$ 6$ y $10 \$$ a la semana en la compra de carne de res, mientras que las compras de las familias de más de 3 miembros, llegan a un $36,8 \%$ en lo que se refiere a las compras de entre $11 \$$ y $15 \$$ a la semana.

Para el análisis se emplearon métodos descriptivos univariantes con la utilización de frecuencias y medidas de tendencia central.

\section{Conclusión}

En lo que concierne el consumo de carne roja en las familias del cantón Ambato, prevalece la de res sobre la de cerdo. Los resultados del estudio contribuyen a la planificación de los comerciantes de este sector, así como también en el diseño de políticas públicas en áreas de salud y mercado.

\section{Anexo}

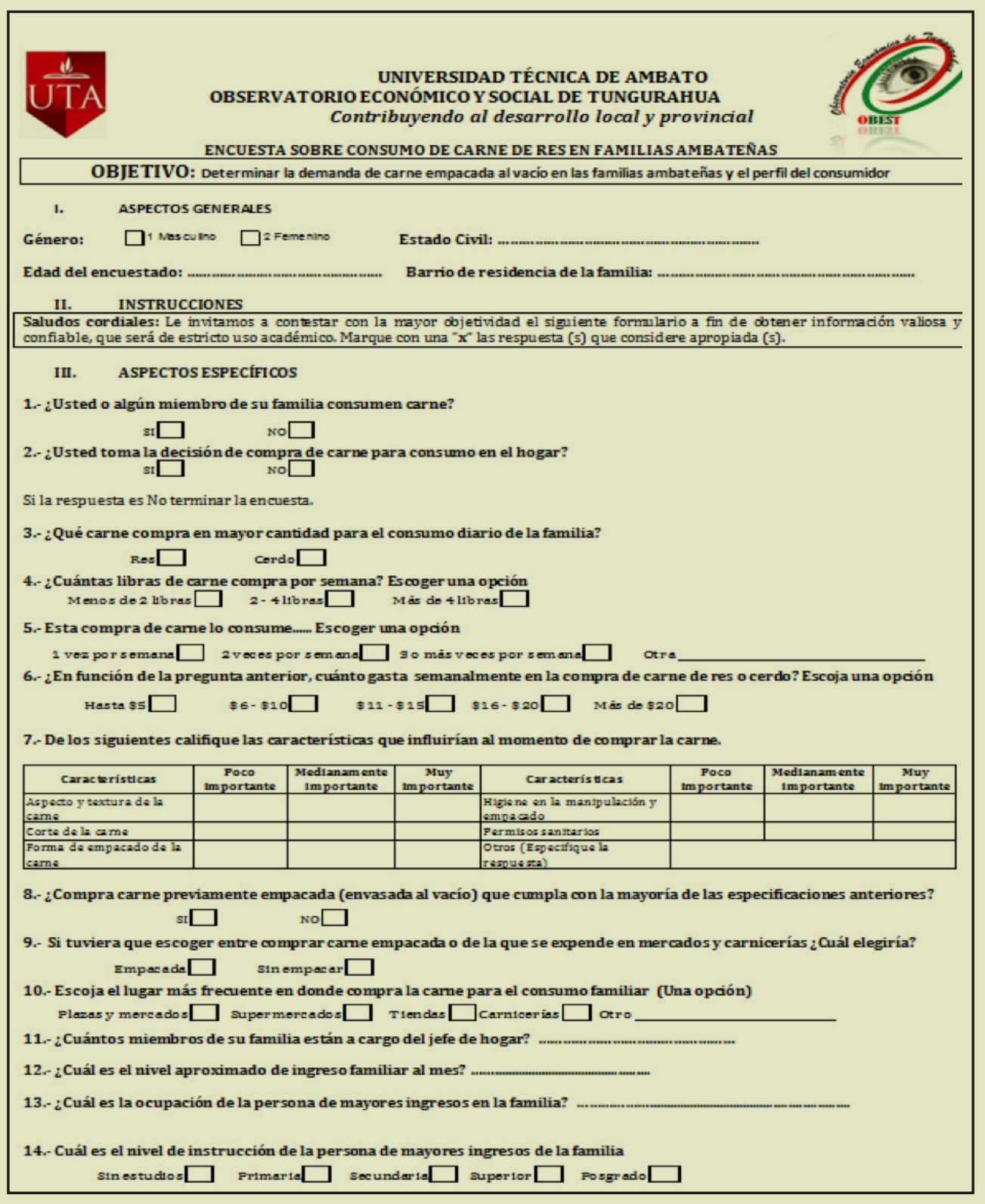

\section{Referencias}

- Instituto Nacional de Estadísticas y Censos. (2016). Promedio de Personas por Hogar, según Cantón. [En línea] Recuperado de:

- http://www.inec.gob.ec/tabulados_CPV/28_Promedio\%20de\%20Personas\%20por\%20Hogar.xls

[Último acceso: 13 noviembre 2016].

- Instituto Nacional de Estadísticas y Censos. (2013). INEC presenta sus proyecciones poblacionales cantonales. [En línea] Recuperado de:

www.ecuadorencifras.gob.ec/inec-presenta-sus-proyecciones-poblaciones-cantonales/

[Último acceso: 16 noviembre 2016].

-Ministerio de Agricultura, Ganadería, Acuacultura y Pesca MAGAP (2015, diciembre). Boletín Agrícola Integral Zona 3. [En línea] Recuperado de:

http://sinagap.agricultura.gob.ec/zonal-integral-zona3/boletin-2015.

[Último acceso: 14 septiembre 2016]. 